\title{
Planning and Operation of Renewable Energy Power System Based on Energy Internet Technology
}

\author{
Zhang Huichao ${ }^{1, a^{*}}$, Wang Zhuo ${ }^{1, b}$, Wang Han ${ }^{1, \mathrm{c}}$ \\ ${ }^{1}$ National Institute of Metrology, China, Changping, Beijing, China
}

\begin{abstract}
Under the leaping development pattern of renewable energy brought by carbon emission targets, large-scale wind and solar power in high proportion of renewable energy power system has brought volatility and uncontrollable factors, and generation-grid coordination has become a technical problem for the safe and stable operation. The concept of energy Internet introduces Internet technology into the field of energy consumption application, which provides a new way for the full coupling of various energy consumption patterns, and is an effective method to solve the problem of safe and stable operation of high proportion renewable energy power system. Based on energy Internet technology, this paper designs the sustainable development architecture of generation-grid coordination from three aspects of planning, operation and mechanism, which lays a solid foundation for the development of generation-grid coordination in the future ' two high ' power system.
\end{abstract}

\section{Introduction}

In the context of carbon emission targets, it is expected that by 2030 , China 's non-fossil energy will account for about 25 per cent of primary energy consumption, and the total installed capacity of wind and solar power generation will exceed 1.2 billion $\mathrm{kW}^{[1]}$. The leaping development of renewable energy will bring about a ' two high ' situation of power system (high proportion of renewable energy and high proportion of power electronic equipment), and the supporting capacity of generation-grid coordination such as frequency, voltage and inertia will become a bottleneck restricting the large-scale consumption of renewable energy.

At present, the three parties of power system, power source, power grid and load are relatively independent, and they are in different forms of planning, operation and mechanism, so it is difficult to form an interoperability situation. The concept of energy Internet is proposed to use Internet technology to guide the full coupling of multienergy grid and energy transport grids ${ }^{[2]}$, and effectively promote the orderly transformation of traditional power systems to high proportion renewable energy power systems. The traditional non-renewable energy such as coal, oil and gas are highly integrated with clean energy such as wind and solar. The different forms of energy use such as electricity and heat are coupled. Through the power grid, the power source and load are interactive through the information cloud, breaking the planning and operation barriers ${ }^{[3]}$, and improving the multi-energy complementary coordination and controllability. Combined with the application of energy Internet technology, this paper puts forward key suggestions to

\footnotetext{
"Corresponding author: ${ }^{2}$ Zhanghc@nim.ac.cn

bwangzhuo@nim.ac.cn

cwanghan@nim.ac.cn
}

promote generation-grid-load-storage coordination from three aspects of planning, operation and mechanism, and designs the development framework to provide key ways for the harmonious development of high proportion renewable energy power system ${ }^{[4]}$.

\section{Forming top-level overall planning and promoting development of generation- grid coordination}

During the 13th Five-Year Plan period, China ' s wind and solar installed power-generating capacity ushered in explosive growth, which broke through the initial planning objectives, but also brought a relatively serious problem of renewable energy abandonment. On the whole, the reasons for power abandonment mainly include two aspects: insufficient peaking capability and insufficient power grid delivery capacity. The reason is that the power source and power grid fail to form a top-level overall planning, and there are technical bottlenecks in the development of generation-grid coordination ${ }^{[5]}$.

The lack of peak regulation capacity is mainly reflected in the failure of coordinated planning between multiple types of power sources. Traditional thermal power planning is mainly related to regional economic growth, with flexible adjustment and stable operation characteristics but belongs to high carbon emission power source; Wind and solar power is a green low-carbon energy, but its output is random and volatile. Due to the limitation of China 's wind and solar resource endowment, it is mostly distributed in the northeast and northwest regions, and has a reverse layout with the load center in the southeast. There are many limitations in power 
consumption. Hydropower is a flexible and controllable power source, but its construction conditions are greatly restricted by water sources and geography. Each type of power source has its own advantages and disadvantages. It is necessary to form a scientific overall planning to meet the regulation needs of high proportion renewable energy power system in the power generation side.

The insufficient transmission capacity of power grid is mainly reflected in the failure of overall planning among power source, power grid and users. The overall power flow in China presents the pattern of North-to-South power transmission and West-to-East power transmission. The energy bases in Northeast and Northwest China, as the sending end, are transmitted to the central and Southeast load center through the UHV AC / DC channel constructed on the grid side. The long-distance transmission of renewable energy brings a series of problems to the sending and receiving end. On the one hand, the energy base at the sending end requires the construction of more supporting traditional power sources, which brings the problem of excess traditional power sources. On the other hand, there is a security problem caused by the large-capacity power input at the receiving end, and there is a low utilization rate of the sending channel at the energy base on the grid side.

Based on the concept of energy internet, this paper proposes a bi-level overall planning architecture for high proportion renewable energy power system, including the lower layer power source planning and the top layer generation-grid-load-storage planning, which provides an effective solution for the development of generation-grid coordination. Firstly, in the power planning layer, the Internet technology is used to connect a variety of power forms with different adjustment capabilities and spatial distribution. The adjustment capabilities and spatial layout of various power sources are fully considered. Combined with the demand of the sending end and the planning of the transmission grid, the capacity and composition of various power sources are designed to promote the generation-grid coordination from the generation side. Secondly, in the overall planning layer of the generationgrid-load-storage overall coordination, the primary energy, power transmission and distribution and thermal grid are organically combined to carry out the multi-agent overall planning of the generation-grid-load-storage, including the capacity of the transmission grid and the construction of the sending end and the receiving end, the consumption space of the load center, the economy and security of cross-regional energy using, and other multidimensional complex problems. Breaking the barriers between different subjects, forming a scientific planning in the multi-stakeholder multi-dimensional category, especially excavate the flexibility of the load side, making full use of the elastic space between various energy consumption modes at the energy consumption side, such as the heat storage characteristics of the pipe grid in the process of heat load transmission, giving full play to the complementary characteristics of various energy consumption modes, overall planning and design, and promoting the generation-grid-load-storage coordinated development of high proportion renewable energy power system. Double layer planning and design layer by layer, mutual influence, through multiple rounds of interactive iterative optimization to obtain scientific and reasonable planning and design, the planning and design architecture of this method is shown in Figure 1.

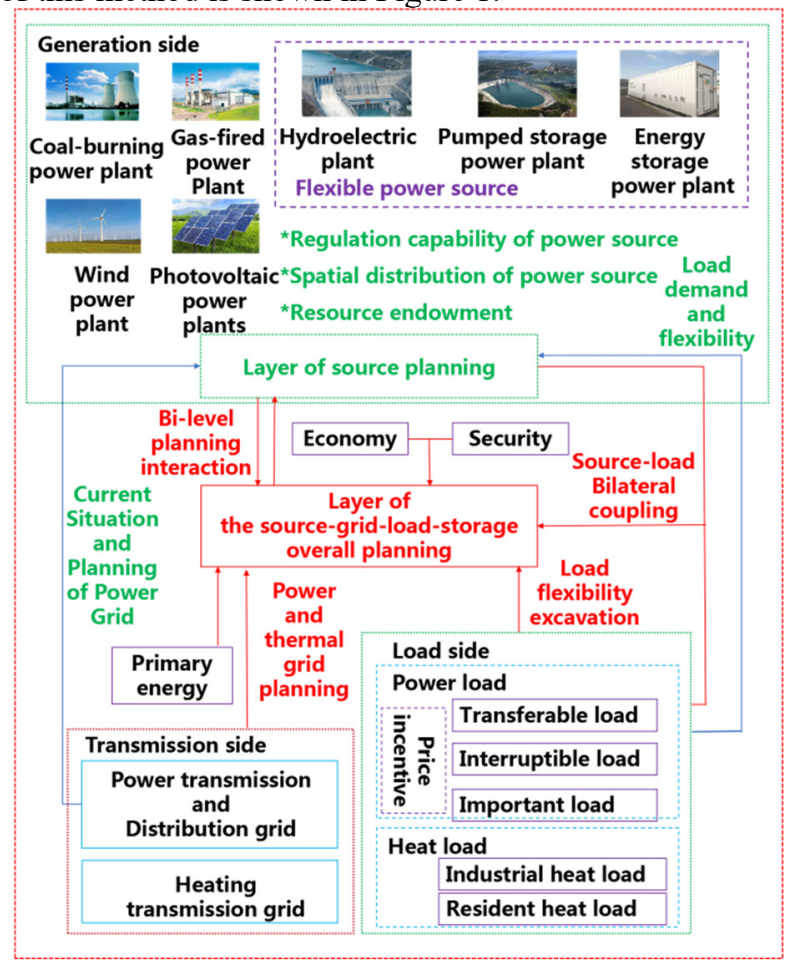

Figure 1. Generation-grid Coordination Planning Architecture Based on Energy Internet Technology

Combined with the development trend of renewable energy in China, two specific suggestions are put forward for the application of bi-level overall planning method of high proportion renewable energy power system in power system planning. On the one hand, for the form of largescale energy base, The total installed capacity of power sources in the base and the proportion of various power sources are comprehensively planned, including highly flexible energy storage power sources. After fully considering the situation of sending and receiving end, economy, security and stability, resource conditions and other multi-dimensional coupling factors on both sides of the source and load, the corresponding planning and design of grid are carried out. On the other hand, for the small-scale park mode, combine the multi-type energy demand of cold, heat and electricity and local new resources, and effectively integrate the characteristics of power grid and thermal grid in the process of load transmission, so as to maximize the coordinated resources of generation-grid-load-storage, and optimize the toplevel planning to realize the multi-energy complementary balance in the local area.

\section{Fully allocating resource operation and improving the generation-grid coordination}

By the end of 2020, the installed capacity of wind and solar power has reached 530 million kilowatts, accounting for $24 \%$ of the total installed capacity of the country. 
Combined with the carbon emission target, it is expected that by 2030 , the total installed capacity of wind and solar power in China will be twice that by the end of 2020. A higher proportion of renewable energy installed and high proportion of power electronic equipment have gradually become the new characteristics of the power system, and also bring greater challenges to the safe and stable operation of the system ${ }^{[6]}$.

In terms of system operation, peak regulation pressure has become a common concern. frequency modulation, fault ride-through, inertia, voltage support and other technical problems have gradually attracted the attention of all parties. As a real-time balance system of power generation, transmission and distribution, the power system is composed of generation side, grid side and load side. The randomness of power consumption at load side is high. The annual load shows the ' two peak ' characteristics of winter peak and summer peak. The load level is relatively low in spring and autumn, and the daily load is mostly daytime two peak characteristics. The load level at night is relatively low, and there is a large peakvalley difference and high peak regulation demand. The proportion of traditional thermal power installed on the power generation side with more emphasis on peak regulation capacity gradually decreases. Unstable characteristics of rapidly developing wind, solar and renewable energy. The peak regulation capacity of the power generation side is relatively tense, and the real-time power balance coordination pressure of the renewable energy power system is increasing. On the other hand, the rapid development of wind and solar power plant are all power electronic equipment. Although the transformation of power generation equipment has been gradually completed in the aspects of high and low voltage fault ride-through and voltage regulation, the ability of frequency modulation and inertia support is weaker than that of traditional thermal power units, and further research is needed in the safe and stable operation of the system.

Based on the concept of energy internet, some scholars have proposed a generalized coordinated and optimized operation mode of generation-grid-load-storage ${ }^{[7]}$, which specifically includes horizontal multi-energy complementarity and vertical generation-grid-loadstorage coordination. They advocate using the resource complementarity among power, oil and gas, and heating systems to complete the substitution and selection of different energy generations. At the same time, they make full use of energy conversion technology and information flow $^{[8]}$, energy flow interaction technology to realize the coordination between resources and grids. For the user side, it is suggested to further expand the demand side management and take various measures to achieve the coordinated and optimized operation of "generation-gridload-storage".

In the operation of power system, in order to improve security and stability, more attention is paid to excavate the regulation ability of power source side, mainly relying on flexible power sources such as thermal power and pumped storage for regulation. There are problems of single control means and limited adjustment space. In order to realize the coordinated control operation of generation-grid-load-storage, the flexibility of using adjustable load should be further explored. With the deepening of energy Internet technology, resource sharing has become a key way for the generation-grid coordination. Information sharing among different subjects of energy generation side, transmission grid and consumption side is the basic premise. By means of digital information technology, resource sharing among different subjects, different times and different regions can be achieved, which forms a virtuous circle pattern of generation-grid-load-storage, and inter-provincial regulation ability. It promotes the generation-grid coordination capacity, and realizes the safe and stable operation of two high power systems. The generation-grid coordinated operation architecture based on energy Internet is shown in Figure 2.

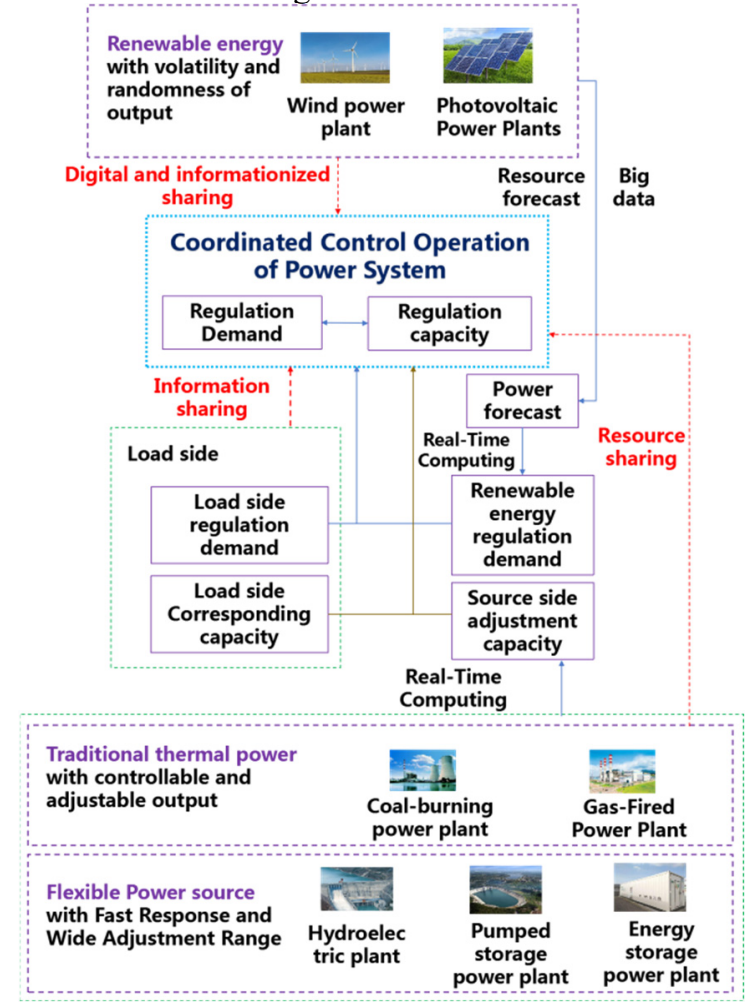

Figure 2. Coordinated Operation Architecture of Generationgrid Based on Energy Internet

\section{Establishing long-term mechanism system to encourage initiative of generation-grid coordination}

In order to improve the level of renewable energy power consumption, conventional thermal power and energy storage power continue to excavate space in peak regulation, frequency regulation and other aspects of grid regulation. In order to maintain the orderly, coordinated and safe operation of multiple types of power sources, and balance the interests of different subjects, various regions have introduced corresponding policy, such as ' two articles ', auxiliary service operation market, power trading market, etc., which effectively promote the generation-grid coordinated development. However, there are still many problems, such as local policy 
inconsistencies, unclear actual implementation, and further improvement of rules formulation. Aiming at the multi-industry, multi-objective and multi-regional differentiation policy of renewable energy, the lack of a unified and long-term mechanism system that can cover multiple dimensions brings some obstacles to power interaction across provinces and regions.

Combined with the thinking of energy internet, in order to promote multi-energy complementary, regional mutual benefit and multi-stakeholder participation in the win-win situation of generation-grid-load-storage coordination, this paper puts forward three suggestions in the policy mechanism: Firstly, in terms of the carrier of the mechanism, it is necessary to give full play to the advantages of multi-energy complementary, strengthen the association of electricity, heat, carbon and other market forms, combine the energy use with the ecological environment closely, and form a full mix of methods and results ; Secondly, in terms of the horizontal application region of the mechanism, in order to avoid the barriers of inter-provincial and inter-regional power transmission and consumption, unified assessment and incentive rules should be established, but not necessarily through the price system to form a comprehensive regional mutual aid of power supply, auxiliary services and security support. Finally, in the aspect of vertical coordination subject, it is necessary to deeply excavate the flexibility of multisubjects of generation-grid-load-storage, fully mobilize the enthusiasm of load side participating in regulation relative to electricity consumption by incentive mechanism, and promote the commercial application mode of energy storage power supply with flexible charging and discharging. At the same time, the assessment mechanism will feedback to the subject to be assessed to actively improve the coordination ability, and finally realize the virtuous circle of multi-stakeholders ' common response and deep participation in coordination.

\section{Conclusion}

In the face of the leaping development of wind and solar renewable energy, the concept of energy Internet puts forward key elements such as integration, interconnection, opening up and sharing. The key to realizing the generation-grid harmonious development lies in the integration of various forms of energy, the interconnection of multi-industry grids, the opening of multi-subjects, and the sharing of multi-class information flow. The formation of an organic coupling unity with resultant force is an effective path to gradually solve the generation-grid coordination problem of high-proportion renewable energy power system.

The practical application of energy Internet technology in power system needs to form the interaction effect of multiple primary energy development modes and energy consumption modes from the planning level, establish the resource sharing situation of Internet technology application from the operation level, and construct an effective incentive mechanism from the policy level to promote the enthusiasm of multiple subjects, so as to form a virtuous cycle of sustainable development pattern.

\section{References}

1. Ma D, Chen W. (2016) Analysis of China's 2030 Carbon Emission Peak Level and Peak Path. China population, resources and environment.26: 1-4

2. Salahuddin M, Alam K. (2015) Internet usage, electricity consumption and economic growth in Australia: a time series evidence. Telematics and Informatics, 32(4): 862-878.

3. Rifkin J. (2011) The third industrial revolution: how lateral power is transforming energy, the economy, and the world. Palgrave MacMillan, New York.

4. Aghaei J, Alizadeh M I. (2013) Demand reponse in smart electricity grids equipped with renewable energy generations: a review. Renewable and Sustainable Energy Reviews, 18: 64-72.

5. Dong Z, Zhao J,Wen F, et al. (2014) From smart grid to energy internet: basic concept and research framework. Automation of Electric Power Systems, 38(15): 1-9

6. Fingrid. (2019) Fast frequency reserve-solution to the nordic inertia challenge. https://www.fingrid.fi/en/pages/news/news/2019/rep ort-fast-frequency-reserve--solution-to-the-nordicinertia-challenge.

7. Zeng M. (2014) Two Key Issues in the Transformation to Clean Energy in China. China Electric Power News. 2014-9-26(1)

8. Tang J, Zhou Z, Niu J, et al. (2014) An energy efficient hierarchical clustering index tree for facilitating timecorrelated region queries in the internet of things. Journal of Network and Computer Applications, 40: $1-11$ 